\title{
Review on the Autonomous Mission Planning System of Satellites
}

\author{
Jianbing Zhu, Jian Guo, Luyuan Wang \\ Institute of Spacecraft System Engineering \\ China Academy of Space Technology \\ Beijing, China \\ e-mail:zhujianbing0103@163.com
}

\begin{abstract}
This The autonomous mission planning becomes a trend to satellites. For this reason, the autonomous mission planning system of satellites is reviewed in this paper. This review can guide the researchers to do more works of the autonomous mission planning system of satellites.
\end{abstract}

Keywords-review; Autonomous Mission Planning; satellites

\section{INTRODUCTION}

Since the 21st century, the aerospace industry in China has stepped into a rapid development period. With the launch of many national major projects such as 'High-resolution earth observation system' and 'Manned spaceflight and moon exploration project' , the traditional remote sensing satellite operation control method that highly depends on ground administration and control has become more and more inadequate for future space mission.

For example, observation plans for remote sensing satellites are pre-made in the ground management and control system, but the enhanced satellite agility in highresolution earth observation system will greatly affect the offline prediction models such as the change of available power on satellites, occupied storage and the influence of meteorological conditions on imaging quality. As a result, ground management and control can not adjust observations timely as real-time environment conditions (clouds, sunlight) change and ground task scheduling can only be implemented conservatively, which can not give full play to satellites, so the obtained observation data can not satisfy users'demand, which has caused a waste of space resources[1]. During deep space exploration activities, the number of time windows between spacecrafts and ground is rather small, but the transmission of telemetry information and remote control data will last for minutes even hours, so it is hard to capture and observe many rare sights only depending on ground control. The above analysis shows that it is the general development trend of Chinese future space industry to strive to develop spacecraft self-management technology.

Self-management aims to reduce satellites' reliability on external environment or even make them independent of external information and control so that remote sensing satellites can make decisions by themselves through sensing themselves and external environment and then complete different tasks. At moment, many institutions such as NASA,
DOD and ESA are devoted to spacecraft self-management and detailed projects are: Deep Space Exploration Project, Traveler (e.g. Mars Traveler), Space-based Observation System (i.e. Hubble Space Telescope) of USA and On-board Autonomy of ESA[2,3,4].

Self-management of remote sensing satellites involves on-board data processing, automated condition monitoring and fault diagnosis, task planning and scheduling among which task planning and scheduling is the nerve center, also the key point of this topic research, that selects actions, allocates resources and breaks down mission tasks into several instruction sequences according to satellite resource status, external environment conditions and other constraints.

During the transition from ground offline management to satellite automated management, remote sensing satellite platform and payload also suffer deep changes so as to satisfy military and civilian remote sensing demands on improving space resolution and time resolution of remote sensing satellites. As a result, remote sensing satellites are gradually featured with high resolution, high agility, rapid response and cooperative networking. In order to give full play to new-type remote sensing satellites, their task planning ability should be improved and extended. The following are analysis and review on the research status of remote sensing satellites and task planning at home and abroad.

\section{FOREIGN SATELLITE SySTEM HAS STEPPED INTO AutOMATED TASK PlanNing}

Wherever Many institutions such as NASA, ESA and CNES are devoted to on-board planning and scheduling so as to support automated operation of spacecrafts and complete complex tasks. Main projects and systems contain: RAX- PS (Remote Agent Experiment-Planning / Scheduling) of NASA, ASPEN (Automated Planning / Scheduling Environment) and CASPER (Continuous Activity Scheduling Planning Execution and Re-planning) responsible for EO-1 satellite control, PROBA (Project for On-Board Autonomy) of ESA and AGATA (Autonomy Generic Architecture - Test and Application) of CNES[2,5,6,7].

a) RAX- PS (Remote Agent Experiment-Planning / Scheduling) of NASA. As part of the automated control system, the main task of RAX-PS is to produce plans that 
can bound all sub-systems according to flight missions. RAX-PS is composed of two parts. One is general planning engine composed of search engine and planning database, the other is specialized knowledge base composed of heuristic function and domain model.

b) ASPEN (Automated Planning / Scheduling Environment) [5] of NASA. ASPEN can generate daily work plan in instructions for EO-1 satellites automatically aimed at more abstract scientific or engineering observation objects. Embedded modeling language of ASPEN can describe main factors in space control field, such as activity, resource, state and parameters, and specific EO-1 domain model (namely the EO-1model established by embedded ASPEN modeling language) mainly needs to model the imaging activity of onboard ALI imaging device on EO-1. Priority of different imaging activities mainly depends on the following factors: cloud coverage in imaging area, solar angle in imaging area, the probability that image data in imaging area can be downloaded before the persistent storage overflows, similarity between EO-1 image and Landsat7 image (phase shift exists between EO-1 and Landsat7 on the same surface) and importance of imaging area.

c) CASPER (Continuous Activity Scheduling Planning Execution and Re-planning) of NASA. CASPER will maintain four parts in each planning: current state, current object, current scheme and belief for future state (deduction and prediction of the system operation track based on the first three parts). Based on fixed time step (as short as seconds) and event step (the moment the next new event occurs), CASPER collects real-time system status and new objects based on which it makes necessary re-planning that is not a newly-made planning scheme but iterate improvement on the current scheme so as to fast generate a feasible scheme satisfying the current state.

d) PROBA (Project for On-Board Autonomy) of ESA. PROBA contains one main load and two additional loads. The main load is a compact high resolution imaging spectrometer (CHRIS) featured with autonomy. Its observation request is object-oriented (for example, an observation request contains the object position and observation duration). The satellite will convert observation request into an instruction about task scheduling, resource decision and satellite pointing. All preparations, commands and data processing related with effective load device operations are planned on-board, including planning, scheduling, resource management, navigation, device pointing and data downloading. Device requirements and planning and scheduling for other activities of spacecrafts are completed by an on-board constraint solving and optimization combination system so as to obtain optimal data. Each task is attached with its own constraints, resource requirements and priority parameter. Besides, cost functions are introduced to optimize long-term planning, and task scheduling is short-term. The aim is to acquire a feasible task sequence.

e) AGATA (Autonomy Generic Architecture - Test and Application) of CNES. The software architecture of AGATA supports the on-board autonomy of spacecrafts in planning, supervision and diagnosis, besides, it needs to be tested and verified. Main objects contain: study on the feasibility of spacecrafts with high autonomy, test on the influence of autonomy on ground operations and operator skills, developing a rapid prototype tool to evaluate the autonomy in future projects, defining and testing the development and verification of autonomy software. The simulation platform is composed of following objects: the space part is composed of one or several spacecrafts and the ground part contains ground station, control center and task center.

\section{FORMATION NETWORKING AND DISTRIBUTED} RUNNING ARE ALSO THE DEVELOPMENT TREND OF FOREIGN REMOTE SENSING SATELLITES

Typical researches are Sensor Web of NASA and TechSat 21 of AFRL[8].

a) Sensor Web of NASA. The sensor web refers to linking ground devices and space devices to complete observation task sets automatically and collaboratively. Observation tasks of sensor web can be triggered by different events for which sensor web will form a special sensor constellation for observation tasks so as to provide detailed observation data as fast as possible. Key techniques contain the event detection technology, monitoring technology for detection data, automated generation of observation requests and automated scheduling of observation tasks. The aim is to acquire data with high time resolution, high space resolution and high spectral resolution.

Current application of sensor web mainly includes floods, vocalic explosion and forest fire monitoring and repeated observation tasks based on clouds monitoring. Involved satellites include MODIS (Terra and Aqua), GOES and EO-1. MODIS is used to detect floods, volcano and forest fire in hot spots, GOES provides nearly real-time clouds coverage information and EO-1 is used to detect hot spots. Once relevant natural disasters and clouds coverage are found, observation tasks in these hot spots will be triggered and act as a trigger source together with observation activities proposed by researchers for ground and on-board task planning and scheduling system which will then convert these observation activities into detailed observation tasks and schedule them scientifically. Observation results will be rapidly reported back to task planning center which will reschedule them upon imaging quality. The whole system is a closed-loop control system.

b) Techsat 21 of AFRL. TechSat 21 (21st century technology satellite in full name) is a distributed space task concept described by AFRL, namely implementing monitoring tasks through the cooperation of multiple satellites in formation flight. The original planned research aims to GMIT (Ground Mobile Target Identification) and SAR (Synthetic Aperture Radar).

Aerospace Corporation Conceptual Design Center has analyzed and verified the performance of small satellite formation through comparing different space-based GMTI concepts. The result indicates that the life cycle cost of small satellite formation has reduced at least by $50 \%$ compared with large satellite system. Besides, the small satellite formation system owns better flexibility and can execute more task types. 
Test flight of TechSat 21 involves a satellite group composed of three small satellites with each weight $150 \mathrm{~kg}$ running on the orbit with $550 \mathrm{~km}$ away from the earth. This satellite group can expand upon requirements aimed at different missions and can reform orbits of small satellites for different task phases to test different task application.

\section{Foreign Remote Sensing SATELLITE WILL BE FEATURED WITH RAPID RESPONSE, HIGH MOBILITY AND DETACHABILITY}

Before Typical researches contain Tactical Satellite Plan of USA, the Hundred Satellite Plan of USA and its allies, F6 Satellite Plan of USA and High-resolution Agile Satellite of western aerospace powers.

a) Tactical Satellite of USA. In order to demonstrate and verify the rapid response space concept and its technique approach and promote the development of quick launch technology, small satellite platform and emergency load technology, USA has proposed and implemented the Tactical Satellite plan (TacSat satellites) as the practice actions for the development of rapid response space technology. TacSat satellites are designed with flexibility, rapid response and affordability, under the control of field commanders, so they can effectively handle the tactical application of space system.

b) Hundred Satellite of USA and its allies. This plan is a huge satellite development and deployment plan aimed at extending the rapid response space concept to the whole NATO, launching and deploying about 100 small tactical satellites in low-earth orbits together with 26 allied countries in NATO. Main function of this plan is: 1) multi-satellite operation in a large satellite group can improve the survivability of the system and stability and robustness of system running. 2) The space-based information network formed by large satellite group networking can form a continuous investigation and log-term monitoring as to a specific ground region, greatly making up defects of current satellites in this aspect. 3) Rapid production, launch and deployment of satellites can improve the power of spacebased information network in rapid repair and rapid recovery, thus enhancing the damage resistance ability and stability of the space military security system. Cost of small tactical satellites in this plan is very low, only 20 million to 30 million dollars. The aim is to confront with the growing stronger counter-space ability of American opponents, build a powerful military alliance with space rapid-response and improve the survivability, tactical application ability and rapid response ability of USA space-based system. At the appointed time, once the USA satellite system is attacked or damaged, USA and its allies can fast recover their space power through this plan.

c) F6 (Future, Fast, Flexible, Fractionated, Free-Flying Spacecraft) plan of USA. The design aim is to develop a future fast flexible fractioned free-flying spacecraft linked with information exchange. Each spacecraft fraction is a small satellite or microsatellite with the weight less than $300 \mathrm{~kg}$. All spacecraft fractions form a distributed structure through multiple launches. The aim is to build a futureoriented, flexible and high-efficiency spacecraft system through decomposing the traditional unitary spacecraft into several composable discrete fractions with different function. These fractions are responsible for different specific tasks, can be quickly manufactured in bulk and be launched independently. They form a complete functional virtual spacecraft system through running collaboratively on orbits with formation running, wireless data transmission and wireless power transmission, which endows the system with system reconfiguration and function redefinition ability throughout the whole life cycle, thus greatly improving the flexibility, extensibility and maintainability of the on-orbit system. Therefore, features of F6 technology can be concluded as: function decomposition, structure separation, wireless linking and formation running.

d) High-resolution Agile Satellite. The development and launches of high-resolution agile satellites, such as USA KH satellite, Russian DK-1 satellite, France Solar-2 satellite, USA WorldView satellites and OrbView-5 satellite[1,3,9], have gradually demonstrated the timeliness requirements of the rapid attitude maneuver of the whole satellite on higher resolution observation tasks and the development trend of current foreign high-resolution satellites that should be more efficient and flexible, can be extended to provide more intelligence information. This kind of satellites are also one approach that can satisfy the urgency and high timelines of users, especially military users, in acquiring high-resolution intelligence information.

\section{SUMMARY}

The autonomous mission planning system of satellites is reviewed in this paper. This review can guide the researchers to do more works of the autonomous mission planning system of satellites.

\section{REFERENCES}

[1] Lamaitre, M.; Verfaillie, G.; Frank, J.; Lachiver, J.;and Bataille, N. 2000. How to manage the new gen-eration of agile earth observation satellites. InPro-ceedings of the International Symposium on Artificial Intelligence, Robotics and Automation in Space.

[2] Globus, A.; Crawford, J.; Lohn, J.; and Pryor, A.2003. Scheduling earth observing satellites with evo-lutionary algorithms. In Conference on Space Mission Challenges for Information Technology (SMC-IT).

[3] Bestaoui Y. Mission plan under uncertainty for an autonomous aircraft. Journal of Aerospace Engineering, 2012, 224(12): 1297-1307

[4] ME Johnson, LM Morre, D Ylvisaker. Minimax and maximum distance designs [J]. Journal of Statistical Planning and Inference, 1990, 26 (2): 131-148.

[5] Chien, S.; Rabideau, G.; Knight, R.; Sherwood, R.;Engelhardt, B.; Mutz, D.; Estlin, T.; Smith, B.;Fisher, F.; Barrett, T.; Stebbins, G.; and Tran, D.2000. Aspen - automating space mission operations using automated planning and scheduling. In SpaceOps 2000, Toulouse, France, June 2000.

[6] D. R. Nelson, D. B. Barber, T. W. McLain, Vector field path following for small unmanned air vehicles, Proc. 2006 American Control Conf, 2006, 5788-5794

[7] R. Vidal, O. Shakernia, H. J. Kim, Multi-agent probabilistic pursuitevasion games with unmanned ground and aerial vehicles, IEEE Trans. Robot. Autom., 2002, 18(5): 662-669

[8] D. Cruz, J. McClintock, B. Perteet, Decentralized cooperative control: A multivehicle platform for research in networked embedded systems, IEEE Contr. Syst. Mag., 2007, 27(3): 58-78 
[9] N. Knoebel, S. Osborne, D. Snyder, Preliminary modeling, control, and trajectory design for miniature autonomous tailsitters, Proc.
AIAA Guidance, Navigation, Control Conf. Exhibit, 2006 